\title{
SIMULATION OF DYNAMIC BEHAVIOR OF QUASI-BRITTLE MATERIALS WITH NEW RATE DEPENDENT DAMAGE MODEL
}

\author{
L. Pereira* ${ }^{\ddagger}$, J. Weerheijm* ${ }^{\dagger}$ and L.J. Sluys* \\ ${ }^{*}$ Delft University of Technology \\ Delft, the Netherlands \\ e-mail: L.F.MagalhaesPereira@TUDelft.nl \\ ${ }^{\dagger}$ TNO Defence, Safety and Security \\ Rijswijk, the Netherlands \\ ${ }^{\ddagger}$ Academia da Força Aérea Portuguesa \\ Sintra, Portugal
}

Key words: Stress-based nonlocal model, Damage, Rate dependency, Dynamic crack-branching

\begin{abstract}
In concrete often complex fracture and fragmentation patterns develop when subjected to high straining loads. The proper simulation of the dynamic cracking process in concrete is crucial for good predictions of the residual bearing capacity of structures in the risk of being exposed to extraordinary events like explosions, high velocity impacts or earthquakes.

As it is well known, concrete is a highly rate dependent material. Experimental and numerical studies indicate that the evolution of damage is governed by complex phenomena taking place simultaneously at different material scales, i.e. micro, meso and macro-scales. Therefore, the constitutive law, and its rate dependency, must be adjusted to the level of representation. For a proper phenomenological (macroscopic) representation of the reality, the constitutive law has to explicitly describe all phenomena taking place at the lower material scales. Macro-scale inertia effects are implicitly simulated by the equation of motion.

In the current paper, dynamic crack propagation and branching is studied with a new rate-dependent stress-based nonlocal damage model. The definition of rate in the constitutive law is changed to account for the inherent meso-scale structural inertia effects. This is accomplished by a new concept of effective rate which governs the dynamic delayed response of the material to variations of the deformation (strain) rate, usually described as micro-inertia effects. The proposed model realistically simulates dynamic crack propagation and crack branching phenomena in concrete.
\end{abstract}

\section{Introduction}

Extraordinary actions such as blast loadings, high velocity impact and earthquakes are rare, but usually have devastating effects. Thus, making critical (infra-)structures, such as powerplants, dams, bridges, hospitals, etc., more resilient is one of the best ways to protect ourselves and our societies from these hazards. Since concrete is a very common construction material, the development of realistic numerical tools to efficiently simulate its failure behavior under extreme loading conditions is of paramount significance.

For example, in case of a high velocity impact of a rigid projectile on a concrete structural element, high rate loads are induced in the target leading to multiple damage modes, such as cratering at the impacted face, spalling 
at the opposite face and radial cracking. Most models used in practical engineering application are used to predict the extension of scabbing and spalling. However, this is clearly insufficient to estimate the integrity and residual bearing capacity of concrete structures where cracking usually spreads over an area much bigger than the crater. Thus, simulating crack initiation, development and branching is essential for a proper representation of dynamic damage development in concrete [1].

Crack branching in brittle and quasi-brittle materials under dynamic loading occurs when crack propagation reaches a critical speed. This limit is usually considered to be material dependent. In brittle materials the critical crack speed is usually set between $40 \%$ and $60 \%$ of the Rayleigh velocity $\left(C_{R}\right)$. For concrete, this limit usually drops to $20 \%$ to $40 \%$ of $C_{R}$ [2]. Furthermore, experimental data (cf. Ravi-Chandra [3]) show that under dynamic loading conditions the evolution and bifurcation of cracks is also highly dependent on the load history and the geometry of the specimen (boundary conditions). Different theories have been developed over the years to explain dynamic fracture of materials, in particular the existence of a critical crack propagation speed and consequent branch phenomena. For example, according to Ravi-Chandar [3], the crack propagation speed is limited by the evolution of the dissipation processes that occurs over a finite fracture process zone (FPZ) around the crack tip involving micro-cracking and other damage mechanisms. Hence, the resistance to crack propagation results in a limited crack speed dependent on the inherent material properties that govern the fracture (damage) process ahead of the crack tip. This concept is particularly appealing for continuum mechanics modeling because, if the fracturing/damage processes are correctly represented by the constitutive law, crack branching phenomena should be automatically simulated without the need for any specific branching criterion. In this contribution we explore this theory and present a new damage model to simulate dynamic fracturing in concrete.
As it is well known, quasi-brittle materials show a highly rate dependent behavior. Although the dynamic behavior of quasi-brittle materials is far from being completely understood, the possible explanations for the observed rate effects are: $i$ ) the enhanced resistance of moisture in the pores [4,5]; ii) the dynamic redistribution of stresses in the fractured zone associated with the material's weakening by micro-cracking; iii) inertia at microlevel which retards the initiation and propagation of micro-cracks [6,7]; and $i v$ ) increase of micro-cracking area (damage) [8,9]. The combined effect of these complex phenomena leads to significant dynamic strength increase [10] associated with a raise of the fracture energy, a visible change of the fracturing patterns and a small (usually neglected) increase of the material stiffness. Furthermore, it has also been observed that the stress-strain relations in a dynamic setting change with a variation of strain rates in time, i.e. the load history [11]. In other words, in case of an abrupt variation of the strain rate, its effects are not 'felt' instantaneously due to micro dynamic (inertia) phenomena. Thus, the common phenomenological rules which consider the dynamic increase factor (DIF) depending on an assumed constant strain rate history are not adequate to describe the dynamic nature of the material. New sound approaches based on physical behavior are needed.

Different damage models have been successfully used to represent crack branching in brittle materials (see for example the work of $\mathrm{Ha}$ and Babaru [12] and Wolff et al. [13]). In this contribution a rate enhanced version of the latest Mazars damage model [14] is used to numerically study dynamic crack branching in concrete. An effective rate is defined in order to account for the dynamic delayed response of the material to variations of the deformation (strain) rate, usually ignored in continuum mechanics. This new parameter is used to update the damage threshold and softening law following a simple damage delay formulation. Finally, a stress-based nonlocal regularization scheme is 
added to the formulation to minimize spurious mesh dependency. After a brief description of the material model, a set of dynamic compact tension tests conducted by Ozbolt et al. [15] is used for validation purposes.

\section{Stress-based nonlocal damage model}

\subsection{Constitutive law}

Continuum damage mechanics has been widely and successfully used to model concrete for a broad range of applications and loading conditions. For the present study, an adapted version of the recently proposed $\mu$ damage model [14] has been developed and implemented in LS-DYNA [16].

A classical definition of isotropic damage is considered. The single scalar variable $\omega$ represents the material's stiffness degradation. The stress tensor is expressed as:

$$
\sigma=(1-\omega) \tilde{\sigma} \quad \text { with } \quad \tilde{\sigma}=C: \varepsilon,
$$

where $\varepsilon$ is the strain tensor, $C$ the elastic stiffness tensor and $\tilde{\sigma}$ is the effective stress tensor [17]. The total amount of damage results from the combination of tensile $\left(\omega_{t}\right)$ and compressive $\left(\omega_{c}\right)$ damage variables following an adapted version of Lee and Fenves's formulation [18].

$$
\omega=1-\left(1-\omega_{t}\right)\left(1-\omega_{c}\right)
$$

The evolution of both damage variables is derived from two principal equivalent strain scalar quantities, $\varepsilon_{t}$ and $\varepsilon_{c}$, which represent the local strain state in tension and compression, respectively

$$
\begin{aligned}
\varepsilon_{t} & =\frac{0.5}{1-2 \nu} I_{\varepsilon}+\frac{0.5}{1+\nu} \sqrt{3 J_{\varepsilon}} \\
\varepsilon_{c} & =\frac{0.2}{1-2 \nu} I_{\varepsilon}+\frac{1.2}{1+\nu} \sqrt{3 J_{\varepsilon}},
\end{aligned}
$$

where $\nu$ is the Poisson's ratio, $I_{\varepsilon}$ is the first invariant of the strain tensor and $J_{\varepsilon}$ the second invariant of the strain deviator tensor, according to:

$$
\begin{gathered}
I_{\varepsilon}=\varepsilon_{1}+\varepsilon_{2}+\varepsilon_{3} \\
J_{\varepsilon}=\frac{1}{6}\left[\left(\varepsilon_{1}-\varepsilon_{2}\right)^{2}+\left(\varepsilon_{2}-\varepsilon_{3}\right)^{2}+\left(\varepsilon_{3}-\varepsilon_{1}\right)^{2}\right]
\end{gathered}
$$

The evolution of the nonlinear response is directly related to the growth of two monotonic internal variables $Y_{t}$ and $Y_{c}$, which account for the historical maximum equivalent strain reached during loading

$$
Y_{i}(t)=\max \left[r_{i} \varepsilon_{i}, Y_{i}(\tau)\right] \text { for all } t \geq \tau
$$

where $r_{t}$ and $r_{c}$ are internal variables derived from the triaxiality factor $(r)$, which provides information on the actual loading state [14, 18]. This triaxiality factor varies between 1 and 0 in tension-compression situations, being 1 in tensile and 0 in compressive conditions

$$
\begin{gathered}
r=\frac{\sum_{I=1}^{3}\left\langle\tilde{\sigma}_{I}\right\rangle}{\sum_{I=1}^{3}\left|\tilde{\sigma}_{I}\right|}, \\
r_{t}=r^{\alpha} \\
r_{c}=(1-r)^{\alpha}
\end{gathered}
$$

where $\left\langle\tilde{\sigma}_{I}\right\rangle$ and $\left|\tilde{\sigma}_{I}\right|$ are the positive and absolute values of the principal effective stresses. The parameter $\alpha$ controls the "amount" of tensile or compressive straining that contributes to the damage evolution in a traction-compression stress-state (see fig. 1). This formulation is simpler than the one used in the original model, but without compromising the representativity of concrete's deviatoric (mixed-mode) response. As it can be seen in fig. 2 both models produce similar 2D failure envelopes, considering $\alpha<0.1$ (black line).

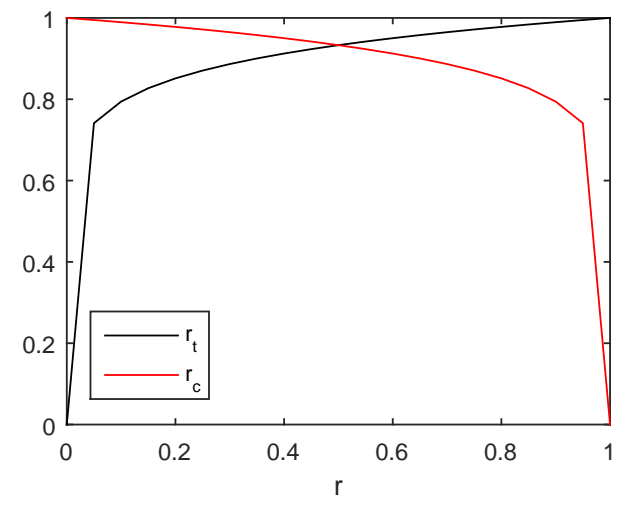

Figure 1: Variation of $r_{t}$ and $r_{c}$ for $\alpha=0.1$

The damage evolution laws for tension $\left(\omega_{t}\right)$ and compression $\left(\omega_{c}\right)$ are of the same type as in 
the original Mazars model [19].

$$
\omega_{i}=1-\left(1-A_{i}\right) \frac{Y_{i 0}}{Y_{i}}-A_{i} e^{-B_{i}\left(Y_{i}-Y_{i 0}\right)}
$$

where $A_{i}$ and $B_{i}$ are material parameters and $Y_{0 i}$ are the equivalent strain damage thresholds. The index $i$ in the equations should be interpreted as $c$ for compression and $t$ for tension.

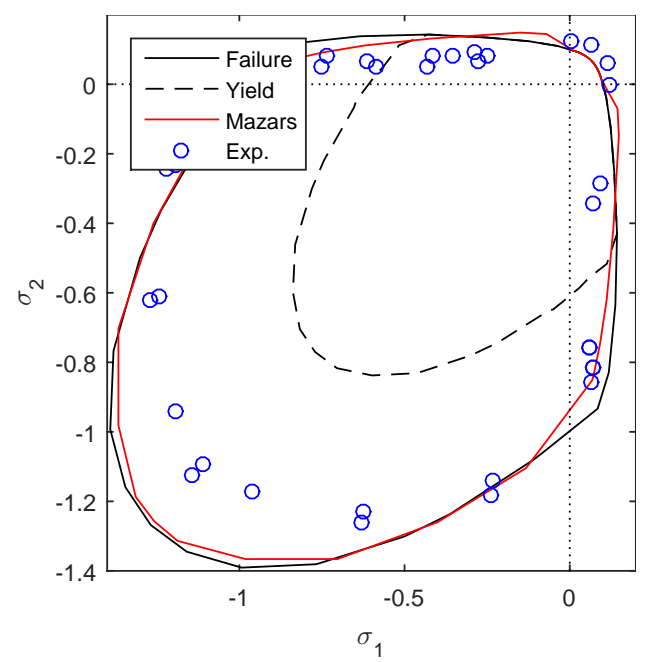

Figure 2: Biaxial failure envelopes ( $\alpha=0.1$ ) compared to Mazars $\mu$ damage model [14] and experimental results by Kupfer et al. [20]

\subsection{Regularization model}

To overcome the spurious mesh sensitivity typical for standard "local" damage models a regularization scheme is needed. In this study, the stress-based nonlocal formulation presented by Giry et al. [21] is used. In this enhanced formulation the nonlocal characteristic length $\left(l_{r}\right)$ is no longer constant. It varies as a function of the stress state of the neighboring elements. The internal length is maximum $\left(l_{r}\right)$ when the material is fully stressed $\left(\sigma_{I}=f_{t}\right)$ and tends to zero when the material is unloaded. To avoid numerical instabilities, a minimum internal length $\left(l_{\min }\right)$ is introduced in the formulation. Consequently, the interactions between elements decrease close to free boundaries, geometrical discontinuities in their normal directions and parallel to damaged areas. This results in a more realistic representation of the evolution of stresses in these situations. For more information about this model and the computation scheme used for this research the reader is referred to [21-23].

\section{Rate-dependent damage model}

It is easily understood that simulating concrete as a combinations of its different phases (aggregates, cement paste and voids) some inherent structural inertia effects being present at meso-scale are naturally taken into account by the equation of motion. For example, the retardation of micro-cracking at high deformation rates associated to micro and meso-scale inertia effects can be analyzed by rate-independent constitutive theories as long as the material is discretized in all its phases [24]. However, in a homogeneous (macro-scale) representation of the material this part of the rate effects has to be explicitly modeled [25]; i.e. the description of rate effects within the constitutive law must be adjusted to the level of representation [6].

Several analytical, experimental and numerical studies demonstrated that the evolution of micro-cracks cannot occur arbitrarily fast due to inertia effects $[26-28]$. Thus, if damage is used to describe microcracking, its evolution has to be retarded in case of high strain rates, i.e. the evolution of damage is a strongly time dependent phenomenon. Moreover, Eibl and Schmidt-Hurtienne [6] suggest that in case of a sudden drop of the strain rate, the effective strength of the material does not decrease instantaneously. The material has some kind of memory and the effects of rate take some time to wear-off, i.e. relax. Consequently it has been suggested that "the dynamic damage evolution requires a strain-history formulation of the dynamic strength instead of the commonly used dependency on the current strain rate in constitutive modeling" (cf. Plotzitza et al. [25]). In other words, in case of an abrupt variation of the strain rate, its effects are not 'felt' instantaneously. Consequently, considering only the current strain state to define an 'instantaneous' rate $(\dot{\varepsilon})$ and derive the corresponding rate effects, as it is usually done, does not adequately describe the complex evolution of stresses in 
dynamics. Here, we propose a new formulation to incorporate in the constitutive law both the relaxation and retardation effects.

\section{Definition of effective rate - $R$}

Different enhanced formulations have been proposed to describe the dynamic damage evolution as a viscous and/or a retardation phenomenon. First, a viscous-damage formulation, similar to a Perzyna-type of viscoplasticity, was used to describe the rate of damage (see for example [29-31]). Plotzitza et al. [25] proposed a formulation where the evolution of damage depends to some extent on the immediately preceding strain history. A history function, derived from a simple Maxwell rheological model, is used to retard the evolution of damage, representing the resistance to micro-cracks growth due to inertia effects. Recently, Haussler-Combe and Kuhn [11] proposed a model to address both viscosity and retarded damage.

Independently of the approach, all these models focused on directly constraining the evolution (i.e. the rate) of damage. In the formulation proposed hereafter, instead of changing the actual damage law, an effective rate $R$ is derived to account for the relaxation and retardation effects. Instead of the typically considered 'instantaneous' strain rate $(\dot{\varepsilon})$, the new parameter $R$ is used to determine the strength and fracture energy in the rate dependent constitutive law, i.e. updating $Y_{0}$ and $B_{t}$ in eq. 9 on the basis of $R$. It is assumed that the effects of a variation of the strain rate take some time $(\lambda)$ to be 'felt'. Simple Maxwell rheological models govern the evolution of the effective rate in case of a decrease or increase of the deformation rate, by the following relaxation and retardation laws, respectively:

$$
\begin{aligned}
& R_{1}=\max \left[\dot{\varepsilon}_{\tau} e^{-\frac{t-\tau}{\lambda}}, \dot{\varepsilon}_{t}\right] \text { for all } t \geq \tau \\
& R_{2}=\min \left[\dot{\varepsilon}_{\tau} e^{\frac{t-\tau}{\lambda}}, \dot{\varepsilon}_{t}\right] \text { for all } t \geq \tau
\end{aligned}
$$

where $\lambda$ is the user defined material's reaction time, $t$ the current time and $\tau$ the time when 'instantaneous' rate was previously constant $\left(\dot{\varepsilon}_{\tau}{ }^{1}\right)$. Please note that both mechanisms are related to inherent dynamic properties of the material, so $\lambda$ may be considered as an intrinsic material property.

To better understand this formulation, let us consider the theoretical examples where a sudden variation of rate takes place before and after damage initiation, represented in fig. 3 by $a$ and $b$, respectively. As it can be seen in fig. 3 in case of a sudden drop of 'instantaneous' rate $\left(\ddot{\varepsilon}_{1}\right)$, the decrease of the effective rate $(R)$ is limited by the relaxation (memory) law. In the completely opposite situation $\left(\ddot{\varepsilon}_{3}\right)$, an increase of effective rate $(R)$ is now limited by the retardation law. This effect is only triggered after damage initiation because it is considered to be related to the resistance to microcracking evolution. Finally, as expected, in case of small or no variation of straining $\left(\ddot{\varepsilon}_{2}\right)$ these dynamic mechanisms do not affect the effective rate $(R=\dot{\varepsilon})$.

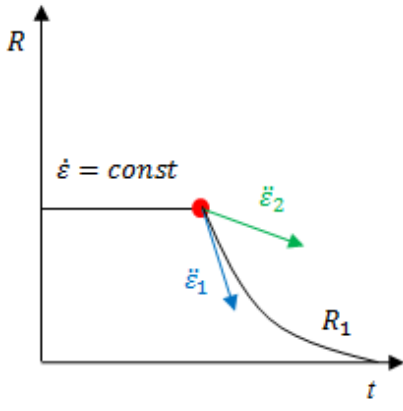

(a)

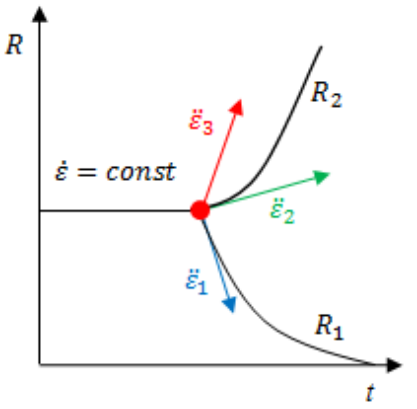

(b)
Figure 3: Maximum allowed evolution of effective rate for: a) $\omega=0$ and b) $\omega>0$

\section{Damage-delay formulation}

The dynamic strength increase is simulated by a simple damage-delay formulation, similar to the one introduced by Pontiroli et al. [32]. In this approach the damage threshold $Y_{t 0}$ is modified as a function of effective rate $(R)$. For ex-

\footnotetext{
${ }^{1}$ In our formulation, strain rate $(\dot{\varepsilon})$ is independently defined for tension and compression as the positive value of the respective equivalent strain $\left(\varepsilon_{t}\right.$ and $\left.\varepsilon_{c}\right)$ variation in time. For example, in tension we have $\dot{\varepsilon}=\left\langle\dot{\varepsilon_{t}}\right\rangle$.
} 
ample, for tension we have:

$$
Y_{t 0}^{d y n}=Y_{t 0}\left(1+\eta R^{n}\right),
$$

where $\eta$ and $n$ are material constants.

The post peak response (softening) of the material associated with the fracturing process is also updated with rate. By changing parameter $B_{t}$ in the damage evolution law (eq. 9), it is possible to control hardening and softening after damage initiation.

$$
B_{t}^{d y n}=B_{t}\left[1-\delta \ln \left(\frac{R}{\dot{Y}_{0}}\right)\right],
$$

where $\delta$ is an input parameter and $\dot{Y}_{0}$ is the rate after which the loading is considered dynamic. Consistently with the $f i b$ recommendation [10] $\dot{Y}_{0}=10^{-6} \mathrm{~s}^{-1}$ is considered.

This evolution of the damage law is meant to describe the variation of fracture energy with rate. It has been experimentally observed that in quasi-static tensile failure conditions, for example, micro-cracks form across the most stressed areas and rapidly coalesce to a main macrocrack which propagates along the weakest regions of the material's matrix. With increasing deformation rates, the concentration of microcracks around the main $\operatorname{crack}(\mathrm{s})$ tend to increase. This phenomenon contributes to an overall increase of the fracture energy represented by eq. 13. It has been experimentally observed that the distribution of these microcracks across the fracture process zone also changes [33]. This phenomenon is indirectly described by the nonlocal length variable used in the regularization scheme. Thus, it is not surprising that calibration of $\delta$ is highly dependent on the material characteristic length.

Fig. 4 shows the variation of the stress-strain relations with rate ( $\dot{\varepsilon}=$ const $)$ for concrete with $f_{t}=3.8 \mathrm{MPa}$, as described in tab. 1. considering $\delta=0.0$ and $\delta=0.045$. The respective peak stresses are presented in the DIF plot in fig. 5 . Analyzing these two figures, one may notice that a variation of $B_{t}^{d y n}$ through $\delta$ has a direct impact on the strain-energy and dynamic maximum strain of the model. Thus, for a proper calibration of the model one has to consider the combined effect of eqs. 12 and 13 , i.e parameters $\eta, n$ and $\delta$.

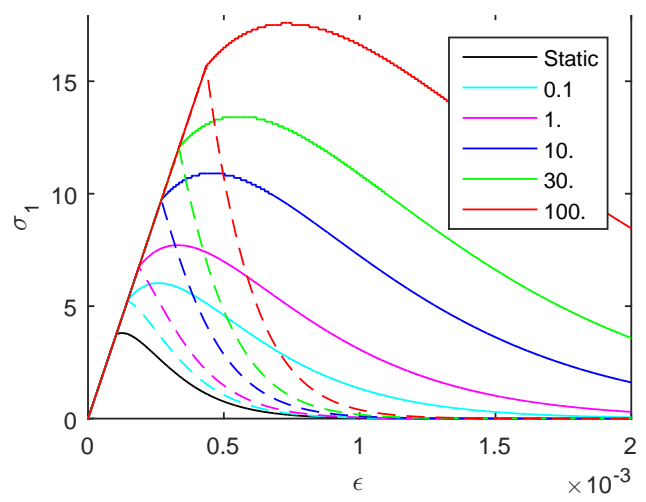

Figure 4: $\sigma-\varepsilon$ relation for different strain rates $(R)$, considering $\delta=0.0$ (dashed) and $\delta=0.045$ (solid).

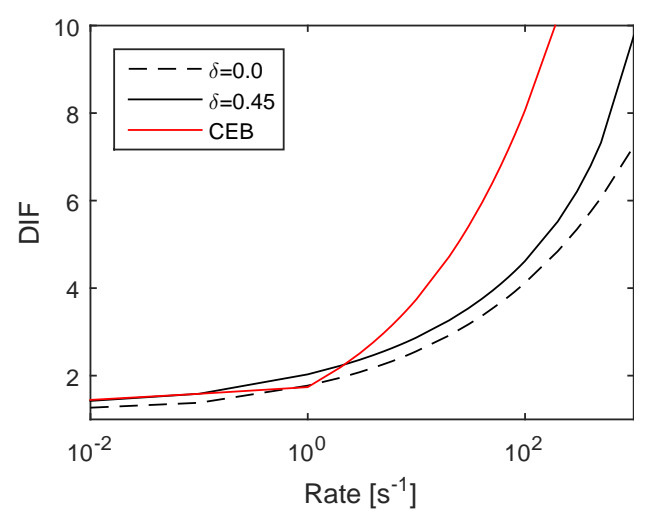

Figure 5: DIF functions.

\section{Simulation of crack branching [15]}

Although studies on dynamic cracking (fracture) are abundant in literature, few are dedicated to study this phenomenon in quasi-brittle materials. Recently, Ozbolt and coworkers conducted two sets of studies, both numerical and experimental, specially designed to study crack branching in plain concrete [15,34-36]. The influence of loading rate on the response of compact tension specimens (CT) and L-specimens of normal strength concrete was investigated. The test results of the CT tests [15] are used as a reference for our study (see fig. 6).

Plain concrete $\left(f_{c}=53 \mathrm{MPa}\right)$ CT specimens $(200 \times 200 \times 25 \mathrm{~mm})$ were tested at different loading rates ranging from 0.06 to $4.3 \mathrm{~m} / \mathrm{s}$. The displacement controlled loads were applied 
at the surface of the right edge of the notch, while the left edge was fixed (see fig. 6). In the experimental study several sensors were used to accurately record, apart of the final crack patterns, the crack propagation speeds, reaction forces and displacement at the notch.

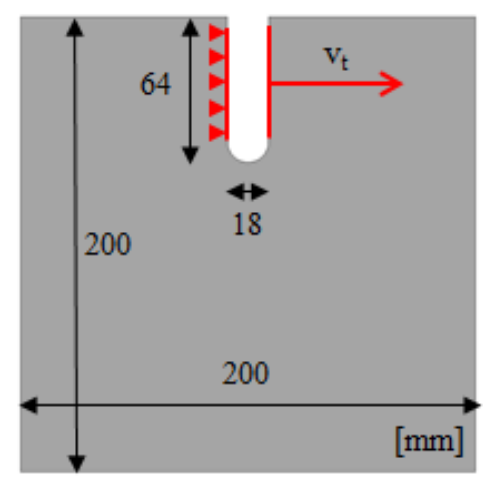

Figure 6: Schematic representation of CT test.

\section{Model description and calibration}

The experiments were simulated with a Lagrangian plane-strain explicit algorithm using the stress-based nonlocal damage model described in section 2. Two structured meshes of quadrilateral element with $2 \mathrm{~mm}$ (M2) and $1 \mathrm{~mm}$ (M1) characteristic element size were used to model the specimens. Displacement controlled loads, similar to the experimentally measured deformation rates, were applied to one edge of the specimen's notch, while the other edge was fixed. The quasi-static properties determined during the experimental campaign [15] were used for the basic calibration of the model (tab. 1).

Since there is no direct relation between fracture energy $\left(G_{F}\right)$ and strain energy in nonlocal damage mechanics, the shape of the softening law (eq. 9) was calibrated considering the average behavior of plain concrete. The parameters in the rate dependent law ( $\eta, n$ and $\delta$ ) were calibrated to fit the simulated experiments. The uniaxial tensile stress-strain relations produced by this model in a single element test for different loading rates and the resulting dynamic increase factors $\left(D I F=f_{t}^{d y n} / f_{t}^{\text {stat }}\right)$ are presented in figs. 4 and 5 , respectively. Finally, the max- imum and minimum nonlocal intrinsic lengths were set as $l_{r}=12 \mathrm{~mm}$ and $l_{\min }=3 \mathrm{~mm}$, considering the tensile equivalent strain as nonlocal variable $\left(\bar{\varepsilon}_{t}\right)$. The calibration of these parameters is not a trivial task and is subject for a separate ongoing study, and therefore not discussed in this paper. Notwithstanding, convergent results were obtained for the stress-based nonlocal formulation with $l_{r} \in[12,16] \mathrm{mm}$. $l_{\text {min }}$ was calibrated as a function of the minimum mesh size to avoid numerical instabilities [21].

Finally, since an explicit computation scheme is used, a sensitivity study was conducted to determine the minimal time-step. It was determined that $30 \%$ (used in this study) or less of the critical time-step ensures numerical stability. In the following section we compare the simulations with the experimental results.

Table 1: Constitutive law parametrization

\begin{tabular}{cc|cc} 
Symbol & Parameter & Symbol & Parameter \\
\hline$E$ & $36 \mathrm{GPa}$ & $\eta$ & 0.80 \\
$\rho$ & $2400 \mathrm{~kg} / \mathrm{m}^{3}$ & $n$ & 0.30 \\
$\nu$ & 0.18 & $\delta$ & 0.045 \\
$f_{t}$ & $3.8 \mathrm{MPa}$ & $\lambda$ & $1.0 \mu \mathrm{s}$ \\
\hline
\end{tabular}

\section{Comparison of simulations with experiments}

In fig. 7 the simulated damage profiles considering the $2 \mathrm{~mm}$ mesh (M2) are given together with the respective experimentally observed crack patterns. Fig. 8 shows equivalent results, but now considering a finer mesh (M1). The maximum reaction forces, registered at the fixed edge of the compact tension specimen's notch, are summarized in table 2. Finally, the crack propagation velocities for the displacement rates of $4.3 \mathrm{~m} / \mathrm{s}$ are presented in fig. 9 alongside to the respective damage profile. The simulated velocities were determined along the center line of the damage profile (i.e. FPZ) considering a time difference $(\Delta t)$ needed for two consecutive elements (at a distance $d s$ ) to reach a specific damage level. Fig. 9 presents the computed velocities considering the damage levels $\omega=0.4$ (left) and $\omega=0.6$ (right). 

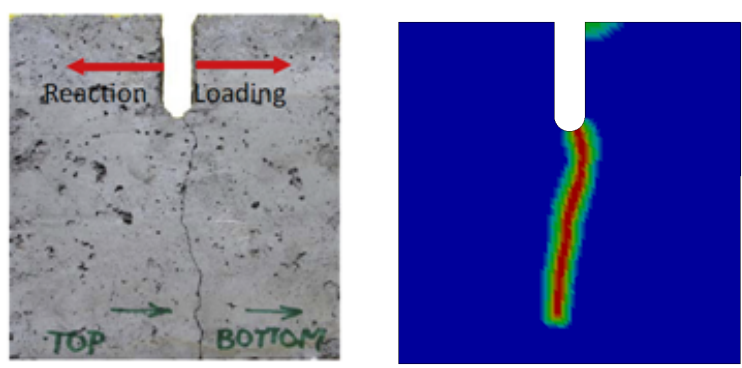

(a)
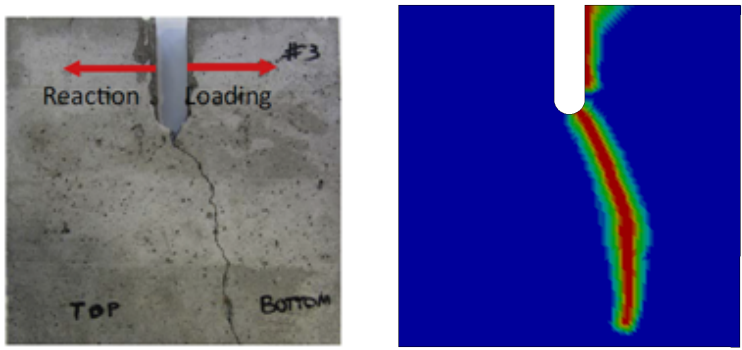

(b)
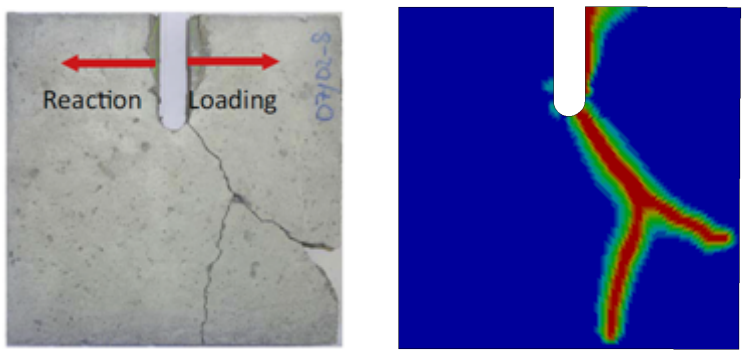

(c)

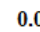

0.5

Figure 7: Comparison between final damage profiles (M2) and experimentally observed crack patterns for different displacement rates:

a) 0.5 ; b) 1.4 and c) $3.3 \mathrm{~m} / \mathrm{s}$

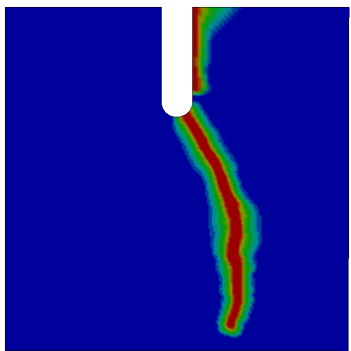

(a)

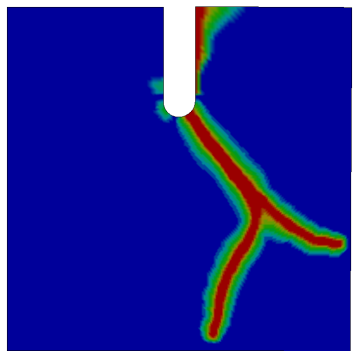

(b)
0.0 0.5

Figure 8: Final damage profiles considering M1 for displacement rates: a) 1.4 and b) $3.3 \mathrm{~m} / \mathrm{s}$

As observed in the experiments, the predicted crack patterns and reaction forces change with loading rate. For loading with a relatively low displacement rate $(0.5 \mathrm{~m} / \mathrm{s})$ damage develops almost perpendicular to the loading direction at relative low propagation speeds. With increasing deformation rates, the damage process accelerates (i.e. crack speed increases) and starts to develop under an inclined angle. In both, simulations and experiments, crack branching is first observed at a displacement rate of $3.3 \mathrm{~m} / \mathrm{s}$. As expected, branching occurs in a region where the damage (crack) propagation speed is the highest, followed by a slight drop in the split cracks. Although the maximum predicted crack speed is slightly higher than the experimentally observed speed before branching, the prediction at the initial stages of cracking are identical (see fig. 9). Overall, the simulations are objective and agree very well with the experimental results. Thus, the dominant dynamic fracture phenomena in concrete are well represented by the proposed damage model.

Table 2: Summary of reaction force for different loading velocities

\begin{tabular}{c|ccc}
\multirow{2}{*}{$\begin{array}{c}\text { Load } \\
(\mathrm{m} / \mathrm{s})\end{array}$} & \multicolumn{3}{|c}{ Max reaction $(k N)$} \\
\cline { 2 - 4 } & Test & M2 & M1 \\
\hline 0.5 & 4.05 & 4.20 & 4.20 \\
1.4 & $4.64-5.76$ & 4.42 & 4.28 \\
3.3 & $4.59-6.88$ & 5.64 & 5.62 \\
4.3 & 5.66 & 5.80 & 5.66 \\
\hline
\end{tabular}

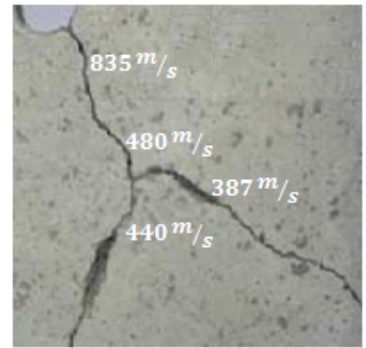

(a)

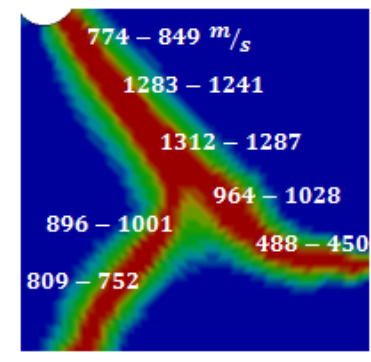

(b)
Figure 9: Crack speed and final damage profile for displacement rate of $4.3 \mathrm{~m} / \mathrm{s}$ 


\section{Critical evaluation of crack branching}

In this section the simulated results are further studied in order to shed some light on the origins of crack branching. Fig. 10 shows the evolution of the principal stresses for deformation rates of 1.4 and $3.3 \mathrm{~m} / \mathrm{s}$, respectively, in the left and right columns.
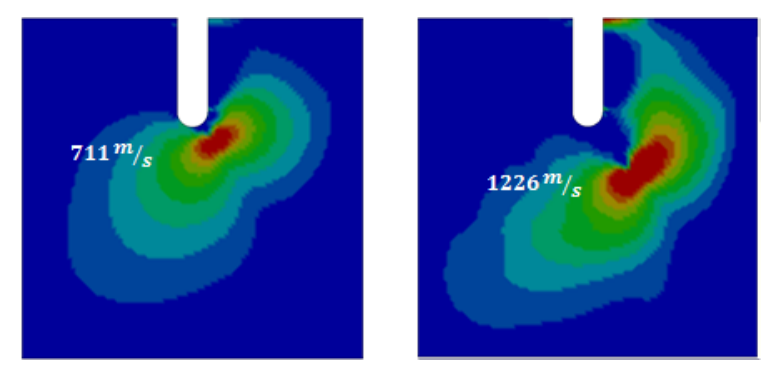

(a)
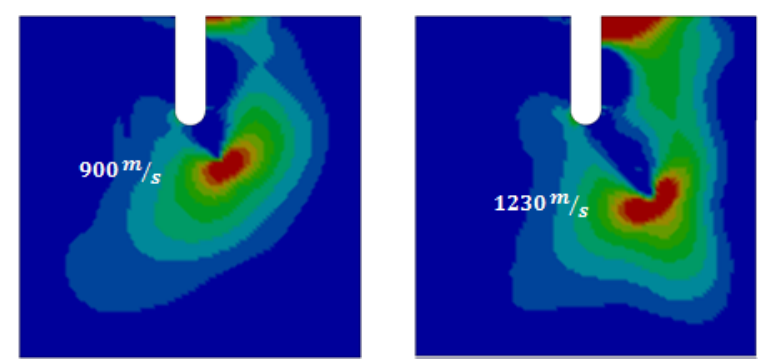

(b)
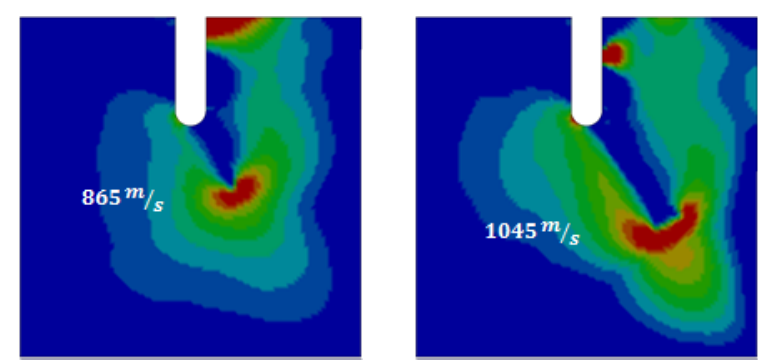

(c)

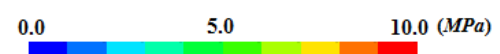

Figure 10: Evolution of principal stresses and crack speed (computed for $\omega=0.5$ )

for $v=1.4 \mathrm{~m} / \mathrm{s}$ (left) and $v=3.3 \mathrm{~m} / \mathrm{s}$ (right).

a) $t=80 \mu \mathrm{s}$; b) $t=100 \mu \mathrm{s}$ and c) $t=120 \mu \mathrm{s}$

It is clear from these images that the principal stress field at the crack tip evolves in time, changing from pure mode-I to mixed-mode. The rate of this change is directly related to the displacement rate the material is subjected to and the crack propagation speed. It is evident in the higher loading rate case (right col- umn fig. 10, that the stress field evolves from a peanut-like shape in the beginning of the failure process to a clover-like shape at crack branching. Since cracking develops perpendicularly to the principal stress direction, this change in the stress field creates the necessary instability condition that leads to crack branching. The combined effect of the specimen's geometry (boundary conditions) with the loading history dictates the final shape of the crack. This is particularly evident in the lower loading rate example (left column fig. 10p were the asymmetric evolution of the stress field makes the crack curve.

It is worth noting that all these phenomena are in good agreement with theoretical predictions and experimental evidences (see for example [37-39]). According to Ravi-Chandar [3], the observed evolution of the principal stress field is a consequence of the evolution of damage in the FPZ. The associated degradation of the effective Rayleigh wave speed in the material surrounding the crack tip, naturally represented by the used damage model [40], is the most probable cause for the observed evolution of stresses. This observation supports the idea that crack branching is governed by the evolution of damage ahead of the crack tip, as suggested by Ravi-Chandra [3], and not by the material's characteristic wave speeds, such as the Rayleigh velocity, or structural inertia effects.

Finally, in order to study the impact of how rate effects are simulated in the overall response of the material, we repeated the simulations considering $a$ ) a rate independent constitutive law and $b$ ) a variation of the rate dependent law where the micro-inertia effects are excluded $(R=\dot{\varepsilon})$. As we can see in figs. 11 and 12 , both models cannot represent the experimentally observed crack patterns (see fig. 7).

When rate effects are excluded (fig. 11) crack branching occurs at lower loading rates simply because the dynamic variation of strength and energy are not modeled, so the material is simply weaker (see fig. 4). In the other case an excessive damage development is observed. This is the consequence of ignoring 
the dynamic resistance to micro-cracking evolution, i.e. ignoring the retardation effect (eq. 11). When this effect is not explicitly simulated, after damage initiation the material starts to soften rapidly leading to a localized straining and consequent increase of strain rate. Since in this case the 'instantaneous' rate $(\dot{\varepsilon})$ is considered instead of effective rate $(R)$ in eqs. 12 and 13 , the variation of $\dot{\varepsilon}$ has a direct impact in the constitutive law.

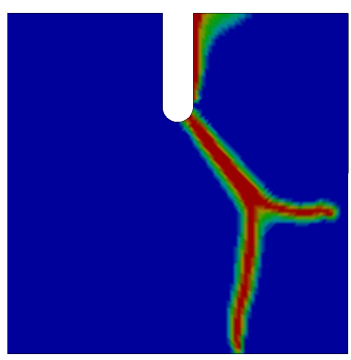

(a)

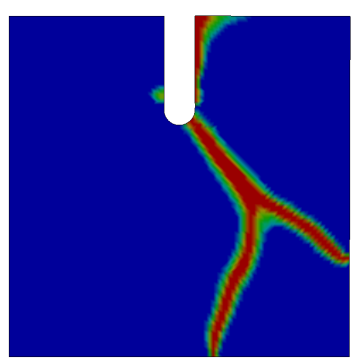

(b)

0.0

0.5

Figure 11: Final damage profiles excluding rate from constitutive law for the loading rates:

a) $0.5 \mathrm{~m} / \mathrm{s}$ and b) $1.4 \mathrm{~m} / \mathrm{s}$

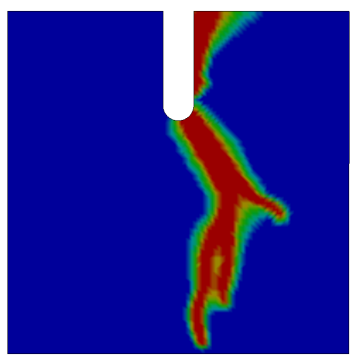

(a)

0.0

$0.0 \quad 0.5$

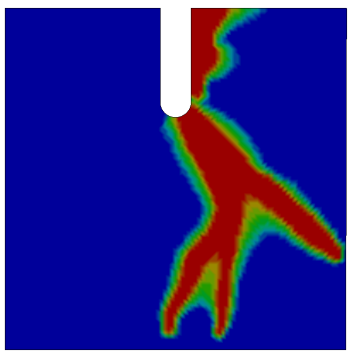

(b)

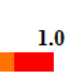

Figure 12: Final damage profiles excluding material's reaction time $(\lambda=0)$, for the loading rates: a) $0.5 \mathrm{~m} / \mathrm{s}$ and b) $1.4 \mathrm{~m} / \mathrm{s}$

\section{Conclusions}

In this contribution the dynamic propagation and branching of cracks in concrete were investigated using a new rate-dependent stress-based nonlocal damage model. We introduced a new concept of effective rate to explicitly simulate the micro and meso-scale dynamic effects, such as the retardation of microcraking due to inertia forces and the delay response of the material in case of variations of strain rate in time ( $\mathrm{mem}$ ory effect). The model is validated against a set of dynamic compact tension tests conducted by Ozbolt et al. [15]

The simulations are objective and agree very well with the experimental results. All major phenomena associated with dynamic crack propagation and branching observed in the experiments can be captured. Thus, the proposed model simulates the dynamic fracturing of concrete realistically.

The results suggest that the crack branching phenomenon is directly related to a distortion of the principal stress field ahead of the crack tip at a critical crack propagation speed. Thus, crack branching is governed by the evolution of damage ahead of the crack tip.

\section{Acknowledgments}

This research is supported by the grant SFRH/BD/79451/2011 from the Portuguese Fundação para a Ciência e Tecnologia (FCT), Lisbon, Portugal and co-funded by the European Social Fund and by Programa Operacional Potencial Humano (POPH). The contribution of the Portuguese Air Force Academy is also gratefully acknowledged.

\section{REFERENCES}

[1] B. Cox, H. Gao, D. Gross, and D. Rittel. Modern topics and challenges in dynamic fracture. J. Mech. Phys. Solids, 53(3):565-596, 2005.

[2] P. Forquin. An optical correlation technique for characterizing the crack velocity in concrete. Eur. Phys. J. Spec. Top., 206(1):89-95, 2012.

[3] K. Ravi-Chandar. Dynamic fracture of nominally brittle materials. Int. J. Fract., 90(1901):83-102, 1998.

[4] P. Rossi. A physical phenomenon which can explain the mechanical behaviour of 
concrete under high strain rates. Mater. Struct., 24(6):422-424, 1991.

[5] A. Ross, D. Jerome, J. Tedesco, and M. Hughes. Moisture and Strain Rate Effects on Concrete Strength (93-M33). ACI Mater. J., 1996.

[6] J. Eibl and B. Schmidt-Huestienn. Strainrate-sensitive constitutive law for concrete. J. Eng. Mech., 125(12):1411-1420, 1999.

[7] J. Weerheijm. Understanding the tensile properties of concrete. Woodhead Publishing Limited, 2013.

[8] J. Zielinski. Fracture of concrete and mortar under uniaxial impact tensile loading. Doctoral thesis, Delft Univ. of Technology, Delft, The Netherlands., 1982.

[9] K. Kirane, Y. Su, and Z. Bažant. Strain-rate-dependent microplane model for high-rate comminution of concrete under impact based on kinetic energy release theory. Proc. R. Soc. London A Math. Phys. Eng. Sci., 471(2182), oct 2015.

[10] fib Model Code for Concrete Structures 2010. Ernst \& Sohn, 2013.

[11] Ulrich Häussler-Combe and Tino Kühn. Modeling of strain rate effects for concrete with viscoelasticity and retarded damage. Int. J. Impact Eng., 50:17-28, dec 2012.

[12] Y. Ha and F. Bobaru. Studies of dynamic crack propagation and crack branching with peridynamics. Int. J. Fract., 162(12):229-244, 2010.

[13] C. Wolff, N. Richart, and J. Molinari. A non-local continuum damage approach to model dynamic crack branching. Int. J. Numer. Methods Eng., 101(12):933-949, 2015.
[14] J. Mazars, F. Hamon, and S. Grange. A Model to Forecast the Response of Concrete under Severe Loadings the $\mu$ Damage Model. Procedia Mater. Sci., 3:979984, 2014.

[15] J. Ožbolt, J. Bošnjak, and E. Sola. Dynamic fracture of concrete compact tension specimen: Experimental and numerical study. Int. J. Solids Struct., 50(25-26):4270-4278, 2013.

[16] J. Hallquist. LS-DYNA Theory manual. Livermore Software Technology Corporation, Livermore, California, 2006.

[17] J. Lemaitre and J. Chaboche. Mechanics of Solid Materials. Cambridge University. Press, 1990.

[18] J. Lee and G. Fenves. Plastic-Damage Model for Cyclic Loading of Concrete Structures. J. Eng. Mech., 124(8):892900, aug 1998.

[19] J. Mazars and G. Pijaudier-Cabot. Continuum damage theory - application to concrete. J. Eng. Mech., 15(2):345-365, 1989.

[20] H. Kupfer, H. Hilsdorf, and H. Rusch. Behavior of Concrete Under Biaxial Stresses. J. Proc., 66(8):656-666, 1969.

[21] C. Giry, F. Dufour, and J. Mazars. Stressbased nonlocal damage model. Int. J. Solids Struct., 48(25-26):3431-3443, dec 2011.

[22] C. Giry, C. Oliver-Leblond, F. Dufour, and F. Ragueneau. Cracking analysis of reinforced concrete structures. Eur. J. Environ. Civ. Eng., 18(7):1-14, jan 2014.

[23] L. Pereira, J. Weerheijm, and L. Sluys. A new rate-dependent stress-based nonlocal damage model to simulate dynamic tensile failure of quasi-brittle materials. Manuscr. submited Publ., 2015. 
[24] L. Snozzi, A. Caballero, and J. Molinari. Influence of the meso-structure in dynamic fracture simulation of concrete under tensile loading. Cem. Concr. Res., 41(11):1130-1142, 2011.

[25] A. Plotzitza, T. Rabczuk, and J. Eibl. Techniques for Numerical Simulations of Concrete Slabs for Demolishing by Blasting. J. Eng. Mech., 133(May):523-533, 2007.

[26] J. Weerheijm. Concrete under impact tensile loading and lateral compression. $\mathrm{PhD}$ thesis, Delft Univ. of Technology, Delft, 1992.

[27] P. Bischoff and H. Perry. Impact behavior of plain concrete loaded in uniaxial compression. J. Eng. Mech., 1995.

[28] A. Krayani, G. Pijaudier-Cabot, and F. Dufour. Boundary effect on weight function in nonlocal damage model. Eng. Fract. Mech., 76(14):2217-2231, sep 2009.

[29] M. Cervera, J. Oliver, and O. Manzoli. A rate-dependent isotropic damage model for the seismic analysis of concrete dams. Earthq. Eng Struct Dyn., 25(April):9871010, 1996.

[30] J. Dube, G. Pijaudier-cabot, C. Borderie, J F Dubé, G. Pijaudier-cabot, and C Laborderie. Rate Dependent Damage Model for Concrete in Dynamics. ASCE J. Eng. Mech., 122(10):939-947, 1996.

[31] F. Gatuingt and G. Pijaudier-Cabot. Coupled damage and plasticity modelling in transient dynamic analysis of concrete. Int. J. Numer. Anal. Methods Geomech., 26(1):1-24, jan 2002.

[32] C. Pontiroli, A. Rouquand, and E. Canton. Shock wave loading on reinforced concrete plates - experimental results and comparision with explicit damage model prediction. In Fram. 2, pages 1219-1228, 1995.

[33] I. Vegt and J. Weerheijm. Influence of moister on the fracture behaviour of concrete loaded in dynamic tension. In V. Saouma, J. Bolander, and E. Landis, editors, FraMCos-9, 2016.

[34] J. Ožbolt, A. Sharma, and H. Reinhardt. Dynamic fracture of concrete - compact tension specimen. Int. J. Solids Struct., 48(10):1534-1543, 2011.

[35] J. Ožbolt and A. Sharma. Numerical simulation of dynamic fracture of concrete through uniaxial tension and L-specimen. Eng. Fract. Mech., 85:88-102, 2012.

[36] J. Ožbolt, N. Bede, A. Sharma, and U. Mayer. Dynamic fracture of concrete L-specimen: Experimental and numerical study. Eng. Fract. Mech., 148:27-41, 2015.

[37] F Erdogan and G C Sih. On the crack extension in plane loading and transverese shear. J. Basic Eng., 85:519-527, 1963.

[38] R Streit and I Finnie. An experimental investigation of crack-path directional stability. Exp. Mech., 20(1):17-23, 1980.

[39] K. Ravi-Chandar and W. Knauss. An experimental investigation into dynamic fracture: I. Crack initiation and arrest. Int. J. Fract., 25:247-262, 1984.

[40] G. Block, M. Rubin, J. Morris, and J. Berryman. Simulations of dynamic crack propagation in brittle materials using nodal cohesive forces and continuum damage mechanics in the distinct element code LDEC. Int. J. Fract., 144(3):131147, 2007. 